\title{
Itinerant Ferromagnetism in an Atom Trap.
}

\author{
Ilya Berdnikov ${ }^{1,2}$, P. Coleman ${ }^{1}$ and Steven H. Simon ${ }^{2}$ \\ 1 Center for Materials Theory, Rutgers University, Piscataway, New Jersey 08854, USA and \\ 2 Alcatel-Lucent, Bell Labs, Murray Hill, New Jersey 07974, USA
}

(Dated: May 29, 2009)

\begin{abstract}
We propose an experiment to explore the magnetic phase transitions in interacting fermionic Hubbard systems, and describe how to obtain the ferromagnetic phase diagram of itinerant electron systems from these observations. In addition signatures of ferromagnetic correlations in the observed ground states are found: for large trap radii (trap radius $R_{T}>4$, in units of coherence length $\xi$ ), ground states are topological in nature - a "skyrmion" in 2D, and a "hedgehog" in 3D.
\end{abstract}

PACS numbers: 67.85.-d, 67.85.Fg, 71.10.Fd

\section{INTRODUCTION}

The simplest, and best studied model of itinerant ferromagnetism (FM) is the Hubbard model ${ }^{1}$. Shortly after its introduction, Nagaoka and Thouless proved ${ }^{2,3}$ that for an interaction $U$ of infinite strength, doping one hole into a background of spins leads to FM. Yet for more than forty years, the fate of the Thouless-Nagaoka phase in the Hubbard model at finite doping and finite interaction has not been fully resolved. Existing studies include various perturbative ${ }^{4,5,6}$, , variational ${ }^{7,8,9,10}$, slave boson 11,12 , Quantum Monte-Carlo $\stackrel{13.14}{ }$ and DMFT 15 calculations. For example, there is no consensus as to the values of the critical doping $\delta_{c r}(\approx 0.19-0.49)$ below which FM occurs and critical interaction $U_{c r}(\approx 63-77.7$ in units of the hopping amplitude $t$ ). Moreover, the various approaches do not agree on the nature of the transition, first order claimed by some $e^{4,5,11}$ second order by others $13,14,15$.

Experiments on optical lattices, which allow a tunable control of model parameters, offer an interesting opportunity to address these long-standing open questions. This setting was used to study correlations in cold bosonic systems experimentally $\stackrel{16,17}{\underline{n}}$, and could also be applied to superconductivity of fermions. The superconducting transition, however, requires a low entropy state cooled to temperatures far below degeneracy, which still poses a significant challenge. Ferromagnetism offers a particular advantage in this respect, since, at least for the Nagaoka phase at $U=\infty$, the absence of any other scales sets the transition temperature to be a finite fraction of the bandwidth, and entropy at the transition is not a small parameter (of order unity per carrier). A recent experiment on two-component fermionic ${ }^{40} \mathrm{~K}$ in optical lattices has reported a Mott insulator with a maximum achieved $U / t \sim 40, T / T_{F}=0.28$ and initial densities of more than one electron per site $\frac{18}{}$. A FM phase presumably resides near the observed Mott phase, and conservative estimates place it at slightly less than one particle per site, $U / t \sim 100$ and $T / T_{F} \sim 0.1 \frac{9,10,15}{}$. The experimental parameters required in a setup such as 18 are estimated: lattice depth $V_{0} / E_{r} \sim 13-19$, depending on the scattering length $a_{s}$, the coherence length $\xi=\frac{\hbar}{\sqrt{2 m \mu}} \sim 0.2 \mu \mathrm{m}$, and the trap radius $R_{T} \sim 1.6 \mu m$, obtained from the laser waist radius of $160 \mu \mathrm{m}$.

In this paper we explore the possibility of using cold fermion gases to study FM in both 2D and 3D systems. For large trap sizes (radius $R_{T}>4$ coherence lengths $\xi)$ with the constraint of zero total magnetization and filling factor less than unity everywhere in the trap, we find that the "skyrmion" configuration (Fig. 1 (a)) has the lowest energy in $2 \mathrm{D}$, while in $3 \mathrm{D}$, the lowest energy configuration is the "hedgehog" (Fig. 1 (b)). Observing these ground states provides a simple way to map out the FM phase diagram, which would be the first result of its kind. (a)

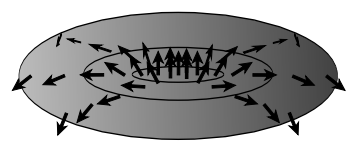

(b)

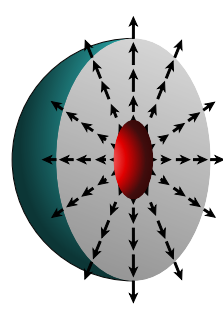

FIG. 1: (Color online) (a)Skyrmion and (b) Hedgehog configuration of spins.

\section{APPROACH}

Our approach to the problem is in marked contrast with previous studies $19,20,21,22$, some of which address only the 2D case: rather than using cold atoms as a means to verify microscopic calculations, we propose using them as a direct probe of itinerant FM in both 2D and $3 \mathrm{D}$ traps. Moreover, in the analysis of 20 total magnetization is not conserved; whereas, in most of the recent experiments ${ }^{23,24,25}$, total magnetization is, in fact, conserved, as a consequence of isolation from the environment and an absence of coupling between the effective spin degree of freedom and the rest of the system.

Ferromagnetism has been studied extensively in the context of multi-component Bose-Einstein condensates 
(spinor BECs), primarily in two dimensions, starting with $26,27,28,29,30,31$. However, magnetic order in these systems is a secondary consequence of condensation, and develops wherever the condensate forms. Fermions near the point of degeneracy, on the other hand, become FM in the absence of any other broken symmetry. Trapping potentials give rise to spatial density variations, so different regions of the fermionic fluid at or near degeneracy may not reach the Stoner instability ${ }^{32}$ simultaneously, resulting in separation between the FM and paramagnetic (PM) phases ${ }^{19}$, something intrinsically absent in spinor BECs and bulk materials. This phase separation ensures that the resulting ferromagnetic droplets have definite size, and this, in turn, allows one to measure the phase diagram, as discussed below.

We consider an optical lattice containing fermionic atoms cooled to temperatures close to degeneracy. The role of spin is played by two states in the atoms' hyperfine multiplet. Hopping between lattice sites is determined by the overlap of the atomic wavefunctions on those sites, while the on-site interaction term is given by the $s$-wave scattering of "electrons" with opposite "spin" 33. In a trapped gas of equal spin populations, ground states are constrained by vanishing magnetization: $\overrightarrow{\mathcal{M}}=\int d \mathbf{r} \vec{M}(\mathbf{r})=0$.

What kind of ferromagnetic quantum state satisfies this condition? One possibility is the state $|\psi\rangle=|S, 0\rangle$ with maximal total $\overrightarrow{\mathcal{M}}^{2}=S(S+1)$, but $\mathcal{M}_{z}=0$. The expectation values of $\mathcal{M}_{x}$ and $\mathcal{M}_{y}$ in this state are zero, ostensibly satisfying the requirement that the total magnetization $\langle\psi|\overrightarrow{\mathcal{M}}| \psi\rangle=0$. However, we can rule out such a state by observing that each component of the total magnetization operator is conserved, $\left[H, \mathcal{M}_{\alpha}\right]=0$, so that the quadrupole operator $\mathcal{Q}_{\alpha \beta}=\mathcal{M}_{\alpha} \mathcal{M}_{\beta}-\frac{1}{3} \mathcal{M}^{2} \delta_{\alpha \beta}$ is also conserved: $\left[H, \mathcal{Q}_{\alpha \beta}\right]=0$. The state $|\psi\rangle$ has a nonvanishing quadrupole moment, where $\left\langle\mathcal{Q}_{x x}\right\rangle=\left\langle\mathcal{Q}_{y y}\right\rangle=$ $-2\langle\mathcal{Q}\rangle_{z z}=S(S+1) / 6$, and therefore cannot develop as a spin-isotropic paramagnetic state is cooled through the ferromagnetic transition.

\section{LANDAU GINZBURG TREATMENT}

To go further, we need to consider states of nonuniform magnetization in which $\overrightarrow{\mathcal{M}}=0$. To this end, we make a long-wavelength expansion of the total energy as a functional of the local magnetization. Such a longwavelength treatment of the problem does not imply that the underlying nature of the system is classical. Indeed, long-wavelength actions of this sort have been used to great success in the analysis of one-dimensional quantum antiferromagnets, where long-range order is completely absent 34 .

The Landau-Ginzburg free energy functional which describes long-wavelength configurations of the magnetiza- tion order parameter takes the form

$$
F_{\mathrm{LG}}=\int d \mathbf{r} \frac{\rho}{2}|\nabla \vec{M}|^{2}+\frac{\beta}{4}\left(|\vec{M}|^{2}+\frac{\alpha(\mathbf{r})}{\beta}\right)^{2}
$$

Odd-power terms are ruled out by the time-reversal symmetry of the free energy. Coefficients $\rho$ and $\beta$ are assumed to be positive and constant for simplicity, and the entire effect of the trap potentials is in the position dependence of $\alpha(\mathbf{r})$. We define $R_{c}$ such that $\alpha(\mathbf{r})<0$ for $r<R_{c}$ and $\alpha(\mathbf{r})>0$ for $r>R_{c}$. We assume that the density everywhere in the system is less than one electron per site, and conclude with a discussion of the remaining cases.

Clearly, whatever magnetic moment is accumulated by any one region in the trap must be completely canceled by the rest of the trap. The configuration which connects any two regions could either be a domain wall or some form of a twist (either with vorticity or without). It is the competition between these three scenarios that determines the ground state. Due to global rotation invariance, it suffices to consider a single representative state from each class. (a)

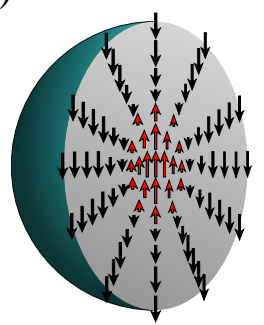

(b)

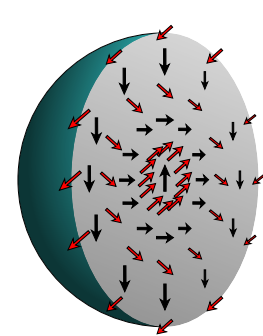

FIG. 2: (Color online) Showing (a) domain wall and (b) single-axis twist configuration of spins

In 3D the first candidate is a hedgehog (Fig. 1] (b)). Qualitatively, it consists of a core of radius $r_{0}$, where magnetization is suppressed, a region of thickness $L$ in which it continuously increases from 0 to some fraction $|a| \leq 1$ of the uniform value $M_{0}$, and the outer region, extending to the edge of the trap at radius $R_{T}=R_{c}$. In a particular realization, the magnetization vector at any point is in the radial direction. A competing configuration has a core of radius $r_{0}$ maximally polarized in one direction. A domain wall of thickness $L$ connects the core to the outer region polarized at a fraction $0 \leq a \leq 1$ of the maximum $M_{0}$ in the opposite direction (Fig. 2 (a)). The remaining possibility is a pure twist. The twist plane is globally fixed (a rotation of $\theta_{0}$ about the $z$-axis), and the magnetization turns about the $x$-axis through an angle $a \pi, 1 \leq a \leq 2$. The twist occupies a shell of thickness $L$ outside of a maximally-polarized core of radius $r_{0}$, and the remaining outer region is polarized along the final direction (Fig. 2) (b)). Parameters $a, r_{0}$, and $L$ for each configuration are determined by numerically minimizing $F_{\mathrm{LG}}$ for a fixed value of $R_{c}$ subject to the constraint of zero net magnetization. 
In $2 \mathrm{D}$ in addition to the domain wall, the single-axis twist and the hedgehog, which are just planar slices of their 3D cousins, there is another possibility: the skyrmion. This configuration has a maximally polarized core of radius $r_{0}$ at the center, a twist through the angle $a \pi, 1 / 2 \leq a \leq 1$ in a ring of thickness $L$, and an outer region, polarized along the final direction (Fig. 1 (a)). Unlike the single axis twist, the twist axis of the skyrmion is a function of position. The constraint for the 2D candidates must be implemented explicitly, and the ground state is obtained the same way as in $3 \mathrm{D}$.

Before solving the full problem, some estimates are in order. In $2 \mathrm{D}$ a domain wall is approximately the suppression of the order parameter in an annulus of thickness $L$ and inner radius $r_{0}$. Omitting a dimensionful prefactor common to all configurations, and in the case of the domain wall, ignoring the stiffness contribution, we find the free energy $(\xi=\sqrt{\rho / \alpha}$ is the coherence length)

$$
F_{\mathrm{DW}} \sim \frac{2 r_{0} L+L^{2}}{2 \xi^{2}}
$$

The energy is minimized by shrinking $L$ to $L \propto \xi$, however, the magnetization constraint forces $r_{0} \propto R_{c}$, and so $F_{\mathrm{DW}} \sim R_{c}$. In the same units the skyrmion free energy has contributions from vorticity and from twisting, i.e.

$$
F_{\mathrm{SK}} \sim 2 \ln \frac{R_{c}}{r_{0}}+(a \pi)^{2}\left(\frac{r_{0}}{L}+\frac{1}{2}\right)
$$

The total magnetic moment of the skyrmion is zero for some $a \sim O(1) . F_{\mathrm{SK}}$ is minimized when both $L, r_{0} \propto R_{c}$. As a result, the skyrmion free energy is roughly independent of the trap size. Analysis of the single axis twist is similar, however, implementing the constraint requires more twisting in the same volume than in the skyrmion case, and therefore this configuration should always be higher in energy. The 2D hedgehog free energy is estimated to be $F_{\mathrm{Hg}} \sim 2 \ln \frac{R_{c}}{r_{0}}+\frac{1}{2}\left(\frac{r_{0}}{\xi}\right)^{2}$, sum of vorticity and core energy, and one can show that $F_{\mathrm{Hg}} \sim \ln R_{c} / \xi$. Thus, for large trap sizes we expect a skyrmion to form in the trap. Similar treatment of the 3D configurations shows that the hedgehog should be the ground state. Numerical computations confirm these estimates, as shown in Fig. 3. We find that in small traps in both $2 \mathrm{D}$ and $3 \mathrm{D}$ domain walls are preferred, while for large traps in 3D $\left(R_{c} / \xi>7\right)$ the hedgehog has the lowest energy, and in $2 \mathrm{D}\left(R_{c} / \xi>4\right)$, the skyrmion is the ground state.

In the above derivation we tacitly assumed that that the whole system becomes FM, i.e. $R_{c}=R_{T}$. This is close to the truth in $2 \mathrm{D}$, since the density of states is practically flat, and the Stoner criterion for magnetic instability dictates that the entire system becomes FM at once, independent of the spatial density variations. In $3 \mathrm{D}$, however, the density of states is more complicated, thus certain parts of the trap will cross into the broken symmetry regime earlier than others. In the language of Landau-Ginzburg, it means that $\alpha$ changes sign from (a)

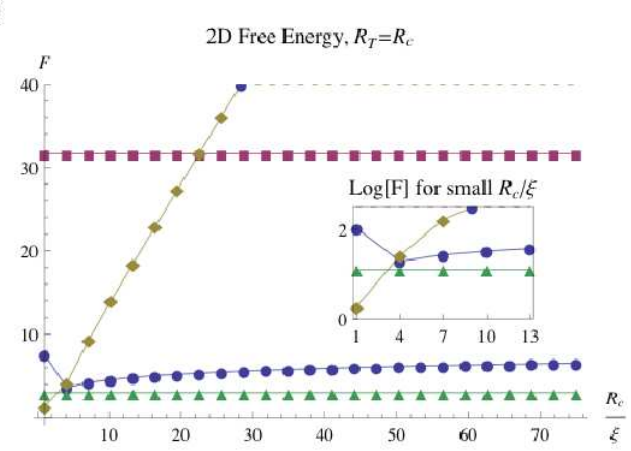

(b)

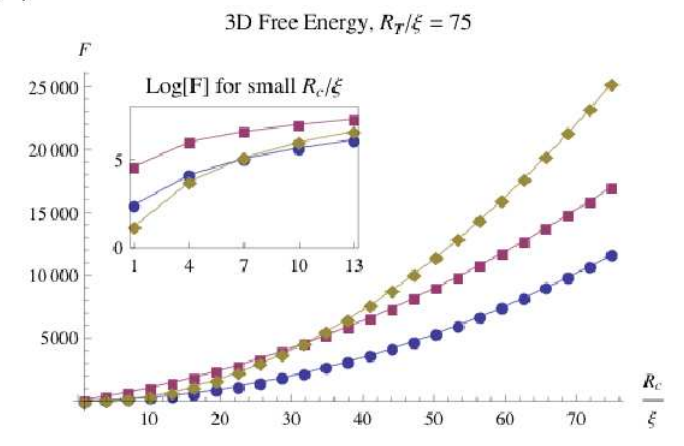

FIG. 3: (Color online) (a) Free energy vs. trap size in two dimensions, $\bullet$ hedgehog, $\boldsymbol{\Delta}$ skyrmion, $\bullet$ domain wall, $\mathbf{a}$ single axis twist. Insert: $\log _{e}(F)$ vs. $R_{c} / \xi$. (b) Free energy vs. size of the correlated region in three dimensions for a fixed trap size, $R_{T}=75 \xi$, $\bullet$ hedgehog, $\bullet$ domain wall, - single axis twist. Insert: $\log _{e}(F)$ vs. $R_{c} / \xi$.

positive outside of some radius $R_{c}$, to negative inside. Incorporating this into our analysis requires somehow suppressing the magnetization outside $R_{c}$ in the candidate states. This can be accomplished by half of a domain wall, e.g. $\vec{M}(r)$ diminishing to 0 within some shell of thickness $L_{1}$ of the critical radius $R_{c}$ and remaining 0 to the edge of the trap $R_{T}$. Although the qualitative results of the calculations remain the same, the numerical problem itself is modified: we minimize $F_{\mathrm{LG}}$ with respect to parameters $a, r_{0}, L$, and $L_{1}$ for a given radius of the FM region $\left(R_{c}\right)$, and a given radius of the trap $\left(R_{T}\right)$. Results for $3 \mathrm{D}$ in Fig. 3 properly reflect these considerations.

In the case when the trap has regions with density higher than one electron per site, we expect further phase separation. In these regions the chemical potential may enter the Mott gap and we would expect an antiferromagnetic Mott phase to set in with filling locked to one electron per site. In regions of even higher density, the chemical potential might cross the gap and emerge, once again, in an itinerant band. Thus, we expect the phase profile to have wedding cake structure $\underline{\underline{17}}$. For traps with very high density in the middle, we expect a hole $\mathrm{PM}$ at the core, followed in turn by shells of hole FM, an anti-ferromagnetic Mott insulator, and an electron FM, finally ending with the electron PM exterior. For inter- 
mediate densities, the Mott insulator could form a natural core for the 3D hedgehog configurations.

\section{DISCUSSION}

Experiments with ultra-cold atomic gases generally exploit absorption imaging to detect the state of matter in the trap. For multi-component gases in-situ imaging of each individual component is possible $23,24,25,35$. In the case of FM systems this technique allows resolving the full magnetization integrated along the camera axis. Fig. 4 shows in-situ images of integrated model magnetization for the hedgehog and the skyrmion. The critical radius can be clearly seen (and measured) in the 3D configuration, and it designates the phase boundary between the PM outside and the FM inside. In addition to the phase boundary and the profile of the magnetization, one could look for signatures of correlation in the shot noise ${ }^{36,37}$. The auto-correlator $\left\langle M_{z}(\mathbf{r}) M_{z}\left(\mathbf{r}^{\prime}\right)\right\rangle$ can be calculated using the time-of-flight images, since it is related to an integral of the frequency space magnetization correlator. Tracking the associated correlation length, which will diverge as the system approaches the transition, is, potentially, a more sensitive tool and should indicate the proximity of the FM phase before the image itself shows the phase separation 31 .

With the newly found signatures of correlation we can map out the phase diagram of an interacting fermion gas. The density of particles at the critical radius $R_{c}$ can be obtained from the absorption images. Properly normalized, e.g. the Mott insulator is exactly at half-filling, this density defines the critical doping of the system. Interaction strength $U / t \sim a_{s} \exp \left(2 \sqrt{V_{0} / E_{r}}\right)$, is given by the $s$-wave scattering length, $a_{s}$ and the lattice depth $V_{0}$, with $a_{s}$ and $V_{0}$ (i.e. $U$ and $t$ ) controlled independently ${ }^{33}$. Thus scanning $a_{s}$ and $V_{0}$ and measuring the critical doping for each, we determine the phase diagram. As discussed above, at higher densities the magnetization images should exhibit an even richer shell structure. However, by particle-hole symmetry all of the FM regions should have the same basic features, and each phase boundary present in the image, will provide a point on the full phase diagram. The finer details of the observed configuration can give us even more details about the parameters of the effective Landau-Ginzburg description; and in turn, our observation of the Landau-Ginzburg pa- rameters can be used to evaluate the efficacy of various microscopic calculational schemes. Similarly, we could explore the vicinity of the FM quantum critical point, and compare the observations to the Hertz-Millis theory. In any real experiment a number of practical issues will play a role. In particular, the details of symmetry breaking must be the same in successive experimental runs and in the different planes of a quasi-2D trap configuration. One way to ensure this is to impose a small position dependent external field. A weak interplanar coupling in quasi-2D lattice might also stabilize the long range order

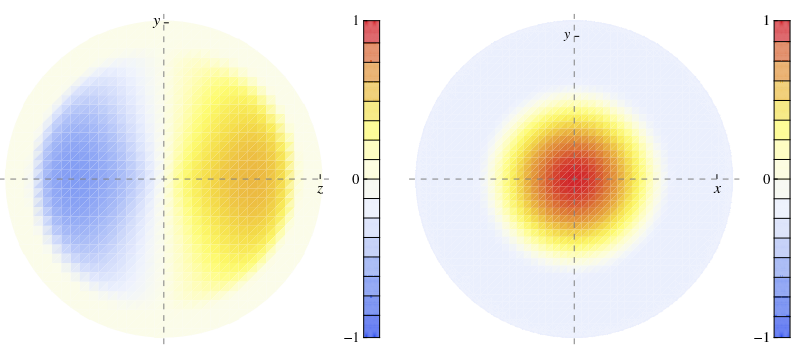

FIG. 4: (Color online) False color plot of magnetization integrated in-situ, symmetry broken the along $z$-axis. Hedgehog imaged along the $x$-axis (left) and skyrmion imaged along the $z$-axis (right).

in the trap. Our model neglects the fact that in experiments to date, strong interactions mix the various bands of the optical lattice 38,39 . Elimination of this effect may require a lower interaction strength, which in turn will require a still lower temperature 33 .

In this paper we demonstrated that ground states of interacting fermions in both 2D and 3D traps are configurations with non-trivial topologies. We've determined detectable signatures of these states, and proposed an experiment to map the phase diagram using these signatures. Such experiments offer the prospect of directly probing itinerant FM, studying the Stoner criterion in strongly correlated magnets, and providing much needed experimental input in the debate on the phase diagram of the Hubbard model.

The authors would like to thank T.-L. Ho and T. Esslinger for fruitful discussions. Supported by NSF Grants No. DMR-0605935, DOE Grant No. DE-FE0200ER45790, and the Rutgers-Lucent Foundation.
1 J. Hubbard, Proc. R. Soc. London A 276, 238 (1963).

2 Y. Nagaoka, Phys. Rev. 147, 392 (1966).

3 D. J. Thouless, Proc. Phys. Soc. London 86, 893 (1965).

4 A. Oles, J. Phys. C: Solid State Phys 15, L1065 (1982).

5 L. B. Ioffe and A. I. Larkin, Phys. Rev. B 37, 5730 (1988).

${ }^{6}$ W. O. Putikka, M. U. Luchini, and M. Ogata, Phys. Rev. Lett. 69, 2288 (1992).

7 B. S. Shastry, H. R. Krishnamurthy, and P. W. Anderson,
Phys. Rev. B 41, 2375 (1990).

8 W. von der Linden and D. M. Edwards, J. Phys. Condens. Matter 3, 4917 (1991).

9 T. Hanisch and E. Müller-Hartmann, Ann. Physik 2, 381 (1993).

10 P. Wurth, G. Uhrig, and E. Müller-Hartmann, Ann. Physik 5, 148 (1996).

11 B. Möller, K. Doll, and R. Frésard, J. Phys. Condens. Mat- 
ter 5, 4847 (1993).

12 D. Boies, F. A. Jackson, and A.-M. S. Tremblay, Int. J. Mod. Phys. B 9, 1001 (1995).

13 X. Y. Zhang, E. Abrahams, and G. Kotliar, Phys. Rev. Lett. 66, 1236 (1991).

14 F. Becca and S. Sorella, Phys. Rev. Lett. 86, 3396 (2001).

15 H. Park, K. Haule, C. A. Marianetti, and G. Kotliar, Phys. Rev. B 77, 035107 (2008).

16 M. Greiner M. Greiner, O. Mandel, T. Esslinger, TW Hänsch and I. Bloch, Nature, 415, 39 (2002).

17 R. B. Diener, Q. Zhou, H. Zhai, and T.-L. Ho, Phys. Rev. Lett. 98, 180404 (2007).

18 R. Jördens, N. Strohmaier, K. Gnter, H. Moritz, T. Esslinger, Nature, 455, 204-207 (2008).

19 L. Salasnich, B. Pozzi, A. Parola, and L. Reatto, J. Phys. B: At. Mol. Opt. Phys. 33, 3943 (2000).

20 T. Sogo and H. Yabu, Phys. Rev. A 66, 043611 (2002).

21 R. A. Duine and A. H. MacDonald, Phys. Rev. Lett. 95, 230403 (2005).

22 Shizong Zhang, Hsiang-hsuan Hung and Congjun $\mathrm{Wu}$, arXiv:0805.3031, to be published (2009).

23 M. Zwierlein, A. Schirotzek, C. H. Schunck and W. Ketterle, Science 311, 492 (2006).

24 G. B. Partridge, W. Li, R. I. Kamar, Y. Liao ad Randall G. Hulet, Science 311, 503 (2006).

${ }^{25}$ Y. Shin, M. W. Zwierlein, C. H. Schunck, A. Schirotzek, and W. Ketterle, Phys. Rev. Lett. 97, 030401 (2006).

26 T.-L. Ho, Phys. Rev. Lett. 81, 742 (1998).

27 T. Ohmi and K. Machida, J. Phys. Soc. Jpn. 67, 1822 (1998).

28 R. A. Battye, N. R. Cooper, and P. M. Sutcliffe, Phys. Rev. Lett. 88, 080401 (2002).

${ }^{29}$ H. Saito and M. Ueda, Phys. Rev. A 72, 023610 (2005).

30 A. E. Leanhardt, Y. Shin, D. Kielpinski, D. E. Pritchard, and W. Ketterle, Phys. Rev. Lett. 90, 140403 (2003).

31 L. E. Sadler, J. M. Higbie, S. R. Leslie, M. Vengalattore and D. M. Stampuer-Kurn, Nature 443, 312315 (2006)

32 E. C. Stoner, Proc. R. Soc. A 165, 372 (1938).

33 I. Bloch, J. Dalibard, and W. Zwerger, Rev. Mod. Phys. 80, 885 (2008).

34 F. D. M. Haldane, Phys. Rev. Lett. 50, 1153 (1983).

35 Y. Shin, C. H. Schunck1, A. Schirotzek and W. Ketterle, Nature 451, 689, (2008).

36 E. Altman, E. Demler, and M. D. Lukin, Phys. Rev. A 70, 013603 (2004).

37 T. Rom, Th. Best, D. Van Oosten, U. Schneider, S. Göllin, B. Paredes and I. Bloch, Nature 444, 733-736 (2006).

38 M. Köhl , H. Moritz, T. Stferle, K. Gnter and T. Esslinger, Phys. Rev. Lett. 94, 080403 (2005).

39 R. B. Diener and T.-L. Ho, Phys. Rev. Lett. 96, 010402 (2006). 